\title{
NEW INEQUALITIES FOR SOME SPECIAL FUNCTIONS VIA THE CAUCHY-BUNIAKOVSKY-SCHWARZ INEQUALITY
}

\author{
CRISTINEL MORTICI
}

\begin{abstract}
We establish new inequalities involving some special functions, using a form of the Cauchy-Buniakovski-Schwarz inequality. Our new inequalities extend the class of the Turan-type inequalites.
\end{abstract}

\section{Introduction}

The well-known Cauchy-Buniakovsky-Schwarz (CBS) inequality states that

$$
\left(\int_{a}^{b} u(t) d t\right)\left(\int_{a}^{b} v(t) d t\right) \geq\left(\int_{a}^{b} u^{1 / 2}(t) v^{1 / 2}(t) d t\right)^{2}
$$

for every functions $u, v:[a, b] \rightarrow[0, \infty)$, such that the integrals does exist.

A. Laforgia and P. Natalini [3] used the following form of the CBS inequality (1.1):

$$
\left(\int_{a}^{b} g(t) f^{m}(t) d t\right)\left(\int_{a}^{b} g(t) f^{n}(t) d t\right) \geq\left(\int_{a}^{b} g(t) f^{\frac{m+n}{2}}(t) d t\right)^{2}
$$

to established some new Turan-type inequalities involving the special functions as gamma, or polygamma functions.

Motivated by this remark, we have the idea to replace $u(t)$ and $v(t)$ in (1.1) by $g(t) f^{m}(t) h^{x}(t)$, respective $g(t) f^{n}(t) h^{y}(t)$, to introduce the following new inequality:

$$
\begin{aligned}
& \left(\int_{a}^{b} g(t) h^{x}(t) f^{m}(t) d t\right)\left(\int_{a}^{b} g(t) h^{y}(t) f^{n}(t) d t\right) \\
& \quad \geq\left(\int_{a}^{b} g(t) h^{\frac{x+y}{2}}(t) f^{\frac{m+n}{2}}(t) d t\right)^{2} .
\end{aligned}
$$

Here, $g, h, f:[a, b] \rightarrow[0, \infty)$ are such that the involved integrals does exist. For $h(t)=1$, or $x=y=0$ in (1.3), we obtain (1.2).

Received January 25, 2010, revised April 29, 2010.

2000 Mathematics Subject Classification. Primary 26D07; Secondary 33B15.

Key words and phrases. Turán-type inequalities, Gamma function, polygamma functions, Riemannzeta function, Cauchy-Schwarz inequality, Hölder inequality. 
The classical Euler gamma function may be defined for $x>0$ by

$$
\Gamma(x)=\int_{0}^{\infty} t^{x-1} e^{-t} d t,
$$

while its logarithmic derivative, denoted

$$
\psi(x)=\frac{d}{d x} \ln \Gamma(x)=\frac{\Gamma^{\prime}(x)}{\Gamma(x)},
$$

is called the digamma function. Its derivatives $\psi^{\prime}, \psi^{\prime \prime}, \psi^{\prime \prime \prime}, \ldots$ are known as polygamma functions.

In the next section, we show how to use the inequality (1.3) to establish new inequalities involving the special functions $\Gamma, \psi, \psi^{\prime}, \psi^{\prime \prime}, \ldots$

\section{The results}

First, by differentiating (1.4), we obtain, for every $n \geq 1$,

$$
\Gamma^{(n)}(x)=\int_{0}^{\infty} e^{-t} t^{x-1} \log ^{n} t d t .
$$

By taking $g(t)=t^{-1} e^{-t}, h(t)=t, f(t)=\log t$ in (1.3), we obtain

$$
\begin{gathered}
\left(\int_{0}^{\infty} t^{-1} e^{-t} t^{x} \log ^{m} t d t\right)\left(\int_{0}^{\infty} t^{-1} e^{-t} t^{y} \log ^{n} t d t\right) \\
\geq\left(\int_{0}^{\infty} t^{-1} e^{-t} t^{\frac{x+y}{2}} \log ^{\frac{m+n}{2}} t d t\right)^{2},
\end{gathered}
$$

which can be rewritten as

Theorem 2.1. For every even integers $m, n \geq 2$, and for every real numbers $x, y \in(0, \infty)$, it holds:

$$
\Gamma^{(m)}(x) \Gamma^{(n)}(y) \geq\left(\Gamma^{\left(\frac{m+n}{2}\right)}\left(\frac{x+y}{2}\right)\right)^{2} .
$$

In particular, for $x=y$, it obtains the Turan-type inequality:

$$
\Gamma^{(m)}(x) \Gamma^{(n)}(x) \geq\left(\Gamma^{\left.\frac{(m+n}{2}\right)}(x)\right)^{2}
$$

The polygamma functions have the following integral representations:

$$
\psi^{(n)}(x)=(-1)^{n+1} \int_{0}^{\infty} \frac{t^{n}}{1-e^{-t}} e^{-t x} d t,
$$

see [1]. By taking $g(t)=1 /\left(1-e^{-t}\right), h(t)=e^{-t}, f(t)=t$ in (1.3), we obtain

$$
\left(\int_{0}^{\infty} \frac{t^{n}}{1-e^{-t}} e^{-t x}\right)\left(\int_{0}^{\infty} \frac{t^{m}}{1-e^{-t}} e^{-t y}\right) \geq\left(\int_{0}^{\infty} \frac{t^{\frac{m+n}{2}}}{1-e^{-t}} e^{-t\left(\frac{x+y}{2}\right)}\right)^{2},
$$

which can be arranged as 
Theorem 2.2. For every integers $m, n \geq 1$ such that $m+n$ is even, and for every real numbers $x$, $y \in(0, \infty)$, it holds:

$$
\psi^{(n)}(x) \psi^{(m)}(y) \geq \psi^{\left(\frac{m+n}{2}\right)}\left(\frac{x+y}{2}\right) .
$$

In particular, for $x=y$, it obtains the Turan-type inequality:

$$
\psi^{(m)}(x) \psi^{(n)}(x) \geq\left(\psi^{\left(\frac{m+n}{2}\right)}(x)\right)^{2} .
$$

Binet's first formula for $\ln \Gamma(x)$ is given by

$$
\log \Gamma(x)=\left(x-\frac{1}{2}\right) \log x-x+\log \sqrt{2 \pi}+\theta(x),
$$

for $x>0$, where the function

$$
\theta(x)=\int_{0}^{\infty}\left(\frac{1}{e^{t}-1}-\frac{1}{t}+\frac{1}{2}\right) \frac{e^{-t x}}{t} d t
$$

is known as the remainder of the Binet first formula for the logarithm of the gamma function. See [1]. By differentiating (2.1), we obtain

$$
\theta^{(n)}(x)=(-1)^{n} \int_{0}^{\infty}\left(\frac{1}{e^{t}-1}-\frac{1}{t}+\frac{1}{2}\right) t^{n-1} e^{-t x} d t .
$$

By taking $g(t)=\frac{1}{t}\left(\frac{1}{e^{t}-1}-\frac{1}{t}+\frac{1}{2}\right), h(t)=e^{-t}, f(t)=t$ in (1.3), we obtain

$$
\begin{aligned}
& \left(\int_{0}^{\infty}\left(\frac{1}{e^{t}-1}-\frac{1}{t}+\frac{1}{2}\right) t^{n-1} e^{-t x} d t\right)\left(\int_{0}^{\infty}\left(\frac{1}{e^{t}-1}-\frac{1}{t}+\frac{1}{2}\right) t^{m-1} e^{-t y} d t\right) \\
& \quad \geq\left(\int_{0}^{\infty}\left(\frac{1}{e^{t}-1}-\frac{1}{t}+\frac{1}{2}\right) t^{\frac{m+n}{2}-1} e^{-t\left(\frac{x+y}{2}\right)} d t\right)^{2},
\end{aligned}
$$

which can be presented as the following

Theorem 2.3. For every integers $m, n \geq 1$ such that $m+n$ is even, and for every real numbers $x$, $y \in(0, \infty)$, it holds:

$$
\theta^{(n)}(x) \theta^{(m)}(y) \geq \theta^{\left(\frac{m+n}{2}\right)}\left(\frac{x+y}{2}\right) .
$$

In particular, for $x=y$, it obtains the Turan-type inequality:

$$
\theta^{(m)}(x) \theta^{(n)}(x) \geq\left(\theta^{\left(\frac{m+n}{2}\right)}(x)\right)^{2} .
$$

The generalized inverse Gaussian distribution is defined for $t>0$ as

$$
g(t)=\frac{1}{I(\alpha ; a, b)} \cdot t^{\alpha-1} e^{-a t-b t^{-1}},
$$


where $a>0, b>0,-\infty<\alpha<\infty$. See [2]. The number $I(\alpha ; a, b)$ is the normalizing constant,

$$
I(\alpha ; a, b)=\int_{0}^{\infty} t^{\alpha-1} e^{-a t-b t^{-\beta}} d t .
$$

By taking $g(t)=t^{\alpha-1}, h(t)=e^{-t}, f(t)=e^{-t^{-1}}$ in (1.3), we obtain

$$
\begin{aligned}
& \left(\int_{0}^{\infty} t^{\alpha-1} e^{-a t-b t^{-1}} d t\right)\left(\int_{0}^{\infty} t^{\alpha-1} e^{-c t-d t^{-1}} d t\right) \\
& \quad \geq\left(\int_{0}^{\infty} t^{\alpha-1} e^{-\frac{a+c}{2} t-\frac{b+d}{2} t^{-1}} d t\right)^{2},
\end{aligned}
$$

which can be written as the following

Theorem 2.4. For every reals $a, b, c, d>0$ and $\alpha \in \mathbb{R}$, it holds:

$$
I(\alpha ; a, b) I(\alpha ; c, d) \geq I^{2}\left(\alpha ; \frac{a+b}{2}, \frac{c+d}{2}\right) .
$$

Now let us introduce the Abramowitz's function [1], defined by

$$
f_{m}(x)=\int_{0}^{\infty} t^{m} e^{-t^{2}-x t^{-1}} d t .
$$

has been used in many fields of physiscs, as the theory of the field of particle and radiation transform.

By taking $g(t)=e^{-t^{2}}, h(t)=e^{-t^{-1}}, f(t)=t$ in (1.3), we obtain

$$
\begin{gathered}
\left(\int_{0}^{\infty} t^{m} e^{-t^{2}-x t^{-1}} d t\right)\left(\int_{0}^{\infty} t^{n} e^{-t^{2}-y t^{-1}} d t\right) \\
\geq\left(\int_{0}^{\infty} t^{\frac{m+n}{2}} e^{-t^{2}-\frac{x+y}{2} t^{-1}} d t\right)^{2},
\end{gathered}
$$

which can be written as

Theorem 2.5. For every positive reals $m, n, x, y$, it holds:

$$
f_{m}(x) f_{n}(y) \geq\left(f_{\frac{m+n}{2}}\left(\frac{x+y}{2}\right)\right)^{2} .
$$

For $x=y$, we obtain the following Turan-type inequality:

$$
f_{m}(x) f_{n}(x) \geq\left(f_{\frac{m+n}{2}}(x)\right)^{2} .
$$




\section{References}

[1] M. Abramowitz and L. A. Stegun (eds.), Handbook of Mathematical Functions with Formulas and Mathematical tables, Dover Publications Inc., New York, 1965.

[2] B. Jorgensen, Statistical Properties of Generalized Inverse Gaussian Distributions, in: Lecture Notes in Statistics, Vol. 9, Springer-Verlag, New York, 1982.

[3] A. Laforgia and P. Natalini, Turán-type inequalities for some special functions, J. Inequal. Pure Appl. Math., 27 (2006), Issue 1, Art. 32.

Valahia University of Târgovişte, Department of Mathematics, Bd. Unirii 18, 130082 Târgovişte, Romania.

E-mail: cmortici@valahia.ro 